\title{
Peer-Induced Fairness in Games
}

\author{
By Teck-Hua Ho and Xuanming Su*
}

\begin{abstract}
People exhibit peer-induced fairness concerns when they look to their peers as a reference to evaluate their endowments. We analyze two independent ultimatum games played sequentially by a leader and two followers. With peer-induced fairness, the second follower is averse to receiving less than the first follower. Using laboratory experimental data, we estimate that peer-induced fairness between followers is 2 times stronger than distributional fairness between leader and follower. Allowing for heterogeneity, we find that 50 percent of subjects are fairness-minded. We discuss how peer-induced fairness might limit price discrimination, account for low variability in CEO compensation, and explain pattern bargaining. (JEL: A12, A13, C72, D63.)
\end{abstract}

Standard theories in economics generate predictions of market behavior by invoking two fundamental assumptions. First, agents are self-interested in that their utility function depends only on their own material payoffs. Second, market behavior is at equilibrium so that no individual agent can achieve a higher payoff by unilaterally deviating from the equilibrium. Recent advances in behavioral economics relax both assumptions by allowing agents, for example, to care about others' payoffs and to make mistakes (see Matthew Rabin, 1998, Colin F. Camerer, George Loewenstein, and Rabin, 2003, and Teck-Hua Ho, Noah Lim, and Camerer, 2006a,b, for comprehensive reviews). This paper focuses on the self-interested assumption and investigates how social comparison may lead to fairness concerns between peers.

A simple and powerful way to demonstrate that people are not purely self-interested is to study the so-called ultimatum game. In this game, a leader and a follower divide a fixed pie. The leader moves first and offers a division of the pie to the follower. The follower can accept or reject. If the follower accepts, the pie is distributed according to the proposal. If the follower rejects, both players earn nothing. When players care only about their own material payoffs, the subgame perfect equilibrium predicts that the leader should offer a small amount (e.g., a dime) to the follower and the follower would accept (since a dime is strictly preferred to nothing). However, data from many experiments (where subjects are motivated by substantial financial incentives) cast doubt on this sharp prediction. Typically, there are almost no offers below 20 percent of the pie. A majority of offers are between 30 percent to 40 percent. Low offers are frequently rejected and the frequency of rejection increases as the offer decreases. These findings are robust to stake size (Robert Slonim and Alvin E. Roth, 1998), persist with repeated trials (Roth et. al, 1991), and prevail across diverse cultures (Joseph Henrich, 2000; Camerer et. al, 2001; Miguel Costa-Gomes and Klaus G. Zauner, 2001).

\footnotetext{
* Ho and Su: Haas School of Business, University of California, Berkeley, CA 94720, Email: Ho: hoteck@haas.berkeley.edu; Su: xuanming@haas.berkeley.edu. Authors are listed in alphabetical order. Direct correspondence to any of the authors. We thank George Akerlof, Eduardo Andrade, Colin Camerer, Vince Crawford, Laura Gardner, Juanjuan Zhang, and three anonymous reviewers for helpful comments. Esther Hwang provided superb research assistance. Taizan Chan designed and developed the software system for running our experiments.
} 
Several solutions have been proposed to resolve this anomaly. These solutions modify a player's utility function by allowing it to depend on the payoffs of other players (for a review see Ernst Fehr and Urst Fischbacher, 2002). In the ultimatum example, each player's utility function now depends on what both players receive. Fehr and Klaus M. Schmidt (1999) propose the so-called "inequity aversion" model in which a player has a disutility of receiving a payoff that is different from the other players. The extent of disutility depends on the player's relative payoff position; players exhibit a stronger disutility from "being behind" than from "being ahead." Gary Charness and Rabin (2002) extend the inequity aversion model to incorporate reciprocity in the utility function (see also Rabin, 1993 and Fehr and Simon Gachter, 2000). This generalized utility function allows players to reciprocate when others have been nice or mean towards them. Gary Bolton and Axel Ockenfels (2000) propose the Equity-Reciprocity-Competition (ERC) model in which each agent's utility function depends on her absolute payoff as well as her relative share of the total payoff. Under ERC, given an absolute payoff, an agent's utility is maximized when her share is equal to the average share. The above models of fairness concerns can be used to capture situations in which agents' social preferences depend on payoffs of other economic agents. We call this distributional fairness concerns.

However, in many real-life situations, people are also driven by social comparison (Leon Festinger, 1954). That is, they have a drive to look to others who are in similar circumstances (i.e., their peers) to evaluate their outcomes and judge whether they have been treated fairly. We term this tendency peer-induced fairness concerns. Indeed, Fehr and Schmidt (1999) suggest that the reference agent for fairness in their inequity aversion model can also come from an external source. Specifically they wrote, "the determination of the relevant reference group and the relevant reference outcome for a given class of individuals is ultimately an empirical question (page 821)." Building on their insight and allowing for different types of reference agent, we distinguish between peer-induced fairness concerns (relative to one's peers) and distributional fairness concerns (relative to other players in the game) and study them simultaneously. We posit that peer-induced fairness concerns can be more salient than distributional fairness concerns when agents engage in social comparison. This is so because social comparison creates a powerful reference point or benchmark for players to compare their well-being with their peer groups.

In this paper, we study peer-induced fairness in a social situation involving 3 economic agents. There are 1 leader and 2 followers. The followers have a similar endowment and the leader plays an ultimatum game with each follower in sequence. Each game involves the leader making a take-it-or-leave-it offer to one of the followers. The two games are identical and independent in that the leader plays the same game with each follower and actions of one game have no bearing on the material payoffs of the other game. However, in between the two games, the second follower obtains an informative but imperfect public signal of the first offer, and uses this signal to infer the first follower's payoff. We analyze this social situation but allow all agents to have distributional fairness concerns and the second follower to have also peer-induced fairness concerns. Our model predicts that if subjects noisily best-respond, the second follower's likelihood of accepting an offer decreases in the signal, suggesting that an identical offer can become less attractive as the second follower's belief of the first follower's payoff increases. In addition, the leader's offer to the second follower is contingent on the signal. The higher the signal, the more attractive the offer will be.

Let us consider three classes of examples of the above game. First, consider a seller that interacts with multiple buyers (e.g., a manufacturer and multiple retailers, a firm and 
multiple customers). Each seller-buyer transaction is independent in that actions within a transaction do not influence material outcomes of other transactions. As distributional fairness would suggest, each individual buyer may care about the seller's payoff in their own respective transaction (in addition to her own material payoff). On the other hand, peer-induced fairness suggests that each individual buyer may also care a lot about what other buyers receive in their interactions with the same seller. For example, a customer cares about what other customers pay for the same product. Similarly, a retailer cares about what contract terms other retailers receive from the same manufacturer. A buyer will treat any unfavorable differences in price or contract terms to be entirely unfair. Second, consider a boss that hires multiple workers with the same skills and performing the same tasks. Clearly, workers care about not only their own wages but also the wages of their peer workers. In fact, bosses often pay their workers a similar wage despite substantial differences in productivity in order to avoid demoralizing less productive workers. ${ }^{1}$ Third, consider a family with multiple children. Sibling rivalry is common and it frequently arises from parents showing favoritism. Clearly, this phenomenon implies that each child's utility function depends also on other children's payoffs. ${ }^{2}$

We test our model's predictions experimentally by engaging subjects in two independent ultimatum games as described above. Using this setup, we find support for our model's predictions. The follower in the second ultimatum game rejects the offer more frequently as the obtained signal increases. The leader's offer is strategic in that she exploits the second follower when the signal is low (even when she has made a good offer to the first follower) and concedes more when the signal is high. In addition, we structurally estimate the model parameters using the data. The estimated peer-induced fairness parameter is 2 times larger than the distributional fairness parameter suggesting that the former is more salient in such social settings. We also incorporate heterogeneity in subjects' taste for fairness by using a latent-class approach. We allow for two different segments, one that is purely self-interested and another that has distributional and peerinduced fairness concerns. Our estimation results suggest that about half the subjects are purely self-interested.

The concept of peer-induced fairness has wide economic implications. We briefly discuss three applications in this paper. First, we show how peer-induced fairness can constrain a monopoly's ability to price discriminate. Without peer comparisons, the monopoly has complete freedom to maximize profits in separate markets that have different economic characteristics. However, when consumers are averse to paying more than their peers, the monopoly may have to narrow price differentials across markets. Second, we show that peer-induced fairness can lead to wage compression. In particular, we show that low variability in CEO compensation packages (see Charles A. O'Reilly, Brian G. Main, and Graef S. Crystal, 1988, for empirical evidence) is necessary in order to prevent dissatisfaction resulting from peer comparisons (i.e., with other CEOs). Third, peer-induced fairness can explain the phenomenon of so-called "pattern bargaining" (see Harold M. Levinson, 1960, Robert C. Marshall and Antonio Merlo, 2004). In many industries, a centralized labor union may negotiate with multiple firms sequentially. Pattern bargaining occurs

\footnotetext{
${ }^{1}$ George A. Akerlof and Janet Yellen (1990) shows that if workers proportionately withdraw their effort because of peer-induced fairness concerns, this behavioral tendency can cause unemployment. Similarly, Fehr, George Kirchsteiger, and Arno Riedl (1993) shows that sellers respond to higher prices from buyers by offering superior quality products.

${ }^{2}$ The sibling rivalry example does not fit our model setup exactly. While our model assumes that there are two independent pies to be negotiated, the two pies in the sibling example may depend on each other. However, the general notion of peer-induced fairness does apply here too.
} 
when the union uses the agreement reached with the first firm as a reference to set the pattern for all subsequent negotiations. Our model naturally explains this phenomenon by peer-induced fairness; any subsequent agreement cannot deviate too much from the outcome of the first negotiation. Pattern bargaining can be problematic when the union and a sequential firm select different reference benchmarks because this difference can restrict the set of feasible negotiation outcomes and lead to labor strikes.

The rest of the paper is organized as follows. The subsequent section formulates the basic model and presents the main equilibrium results. Section II describes the experimental design and procedure. Section III presents the experimental results and calibrates the basic model using the data. Section IV generalizes the basic model by incorporating heterogeneity. Section V describes three economic applications of peer-induced fairness. Section VI concludes.

\section{Basic Model}

\section{A. Model Setup}

There are 3 players - 1 leader and 2 followers. The leader plays an identical ultimatum game with each of the followers in sequence. In each game, there is a fixed pie of size $\pi$ to be divided between the leader and one of the followers. The leader moves first and offers $s_{1}$ to the first follower. The first follower's decision $a_{1}$ can either be to accept $\left(a_{1}=1\right)$ or to reject $\left(a_{1}=0\right)$. If $a_{1}=1$, the leader receives $\pi-s_{1}$ and the follower receives $s_{1}$. Otherwise, both receive 0 . The second follower obtains a signal $z=s_{1}+\epsilon$, where $\epsilon$ is a zero-mean random noise term with an arbitrary distribution function $F(\cdot)$ and density function $f(\cdot)$. Based on this signal, the second follower makes inferences regarding the first offer, and these beliefs influence his decision to accept or reject. The same signal $z$ is observed by the leader before the second game begins. Then, the leader makes an offer $s_{2}$ to the second follower possibly based on the signal $z$. Again, the follower's decision $a_{2}$ can be either to accept $\left(a_{2}=1\right)$ or to reject $\left(a_{2}=0\right)$. If $a_{2}=1$, the leader receives $\pi-s_{2}$ and the follower receives $s_{2}$. Otherwise, both receive nothing. Note that the leader receives material payoff in both games while each of the followers receives material payoff in their respective game.

Let us define the agents' utility functions. Consider the utility function of the first follower $U_{F 1}\left(s_{1}, a_{1}\right)$. The follower's utility function has two components. The first component is the agent's material payoff from the game and the second component reflects the first follower's disutility from receiving a payoff that is behind that of the leader. Hence, the second component captures distributional fairness concerns.

$$
U_{F 1}\left(s_{1}, a_{1}\right)= \begin{cases}s_{1}-\delta \cdot \max \left\{0,\left(\pi-s_{1}\right)-s_{1}\right\}, & \text { if } a_{1}=1 \\ 0, & \text { if } a_{1}=0\end{cases}
$$

Here $\delta$ is the parameter capturing the degree of aversion from being distributionally behind. ${ }^{3}$

\footnotetext{
${ }^{3}$ Our model can be extended to include an additional disutility term resulting from being ahead. This is in the spirit of Charness and Rabin (2002) and Fehr and Schmidt (1999). For example, the first follower's utility function can also include the term $-\delta^{\prime} \cdot \max \left\{0, s_{1}-\left(\pi-s_{1}\right)\right\}$. However, in Table 4 below, additionally allowing for this parameter yields the estimate $\hat{\delta}^{\prime}=0$. Thus distributional fairness concerns associated with being ahead are absent in our experimental data. We chose to use the simplest
} 
The second follower's utility function $U_{F 2}\left(s_{2}, a_{2}\right)$ is defined similarly except that it also contains an additional component. This component arises from the second follower's tendency of comparing herself to a similar peer (i.e., the first follower) and reflects the disutility from being behind. Recall that $z=s_{1}+\epsilon$ is the signal observed by the second follower and the leader. Using the signal $z$, the second follower can infer the probability $\hat{p}(z) \equiv P\left(a_{1}=1 \mid z\right)$ that the first offer is accepted and the conditional expectation of what the first follower receives $\hat{s_{1}}(z) \equiv E\left(s_{1} \mid z, a_{1}=1\right)$. We will discuss the signal inference process in more detail later. For now, let us use $\hat{p}(z)$ and $\hat{s_{1}}(z)$ to denote these inferences. The second follower's utility function is given next:

$$
U_{F 2}\left(s_{2}, a_{2} \mid z\right)=\left\{\begin{array}{cc}
s_{2}-\delta \cdot \max \left\{0,\left(\pi-s_{2}\right)-s_{2}\right\} & \\
-\rho \cdot \hat{p}(z) \cdot \max \left\{0, \hat{s_{1}}(z)-s_{2}\right\} & \text { if } a_{2}=1, \\
0, & \text { if } a_{2}=0
\end{array}\right.
$$

As before, the parameter $\delta$ reflects the second follower's degree of aversion from being distributionally behind the leader. In addition, the parameter $\rho$ represents the degree of aversion from being behind in a social comparison with a peer. ${ }^{4}$ We define "peer" as a reference agent that satisfies two conditions. First, the reference agent must play the same role or position in the game (e.g., another follower). Second, the reference agent must face the same social situation (e.g., both have accepted or both have rejected the offer). In general, the notion of a peer can clearly go beyond these specific contexts. In a clever study using a panel-level dataset, Erzo F. P. Luttmer (2005) shows that a person's self-reported happiness decreases with an increase in neighbors' incomes. Clearly, the neighbors may not have the same employer or even occupation. In our experimental setup, subjects interact anonymously and the task context becomes the only relevant cue for determining who their peer is. Luttmer's finding suggests that other social cues such as geographical location and regular social interactions can be equally compelling as criteria for defining a peer.

Linking the above definition of a peer to the utility function (I.2), there are two cases. The first is when the second follower accepts $\left(a_{2}=1\right)$. In this case, there is probability $\hat{p}(z)$ that the first follower has also accepted. The second follower treats the first follower as a peer and experiences a disutility when what she receives $s_{2}$ is behind what she believes the first follower has received $\hat{s}_{1}(z)$. The second case is when the second follower rejects $\left(a_{2}=0\right)$. In this case, the first follower must also have rejected to qualify as a peer. In this case, both followers get nothing. So the second follower's utility is zero. ${ }^{5}$

possible model to demonstrate the existence of peer-induced fairness because it makes the model more tractable and allows us to generate sharp predictions about subjects' behaviors.

${ }^{4}$ The aversion of being behind is similar to the notion of loss aversion (Daniel Kahnemann and Amos Tversky, 1979; Camerer, 2001). People have a negative transaction utility when receiving a payoff that is below a well-defined reference point. Ronald Bosman and Frans van Winden (2002) show that a follower's expectation of what she is likely to receive serves as a reference point and can significantly influence her acceptance decision in a continuous version of an ultimatum game. The notion of loss aversion has also been applied to a business-to-business channel setting to show why nonlinear pricing contracts may not work as well as the standard models would suggest because these pricing contracts yield a negative transaction utility (Lim and Ho, 2007, and Ho and Zhang, 2008).

${ }^{5}$ It is possible that the second follower, when rejecting, may engage in social comparison with the first follower who has accepted. If this is true, the second follower will experience a negative utility of $-\eta \cdot \hat{p}(z) \cdot \hat{s_{1}}(z)$ from rejecting, since there is a probability $\hat{p}(z)$ that the first follower has accepted and received $\hat{s_{1}}(z)$. Here, we distinguish the parameter $\eta$ from our parameter $\rho$ above because the former involves social comparison with an agent in a different situation. Allowing for $\eta$ in our structural 
The leader receives material payoffs from both ultimatum games. In the second game, the leader receives the utility $U_{L, I I}\left(s_{2}, a_{2} \mid z\right)$ given as:

$$
U_{L, I I}\left(s_{2}, a_{2} \mid z\right)= \begin{cases}\pi-s_{2}-\delta \cdot \max \left\{0, s_{2}-\left(\pi-s_{2}\right)\right\}, & \text { if } a_{2}=1 \\ 0, & \text { if } a_{2}=0\end{cases}
$$

Note that the leader's utility in the second game depends on the signal $z=s_{1}+\epsilon$ insofar as the second follower's decision rests upon it. In the first game, the leader receives the utility $U_{L, I}\left(s_{1}, a_{1}\right)$ as given below:

$$
U_{L, I}\left(s_{1}, a_{1}\right)= \begin{cases}\pi-s_{1}-\delta \cdot \max \left\{0, s_{1}-\left(\pi-s_{1}\right)\right\}, & \text { if } a_{1}=1 \\ 0, & \text { if } a_{1}=0\end{cases}
$$

The general model of Fehr and Schmidt (1999) allows for fairness concerns between all possible pairs of players. Our model builds on their model by allowing two different kinds of fairness for different pairs of players. Specifically, we distinguish fairness concerns between the leader and a follower from fairness concerns between two followers and show that the latter can be more significant when players engage in social comparison.

We can solve the game using the standard backward induction principle. In the second game, the leader chooses $s_{2}$ to maximize $U_{L, I I}\left(s_{2}, a_{2} \mid z\right)$. In the first game, the leader chooses $s_{1}$ to maximize $U_{L, I}\left(s_{1}, a_{1}\right)+U_{L, I I}\left(s_{2}, a_{2} \mid z\right)$.

\section{B. Second Follower's Inferences, $\hat{p}(z)$ and $\hat{s_{1}}(z)$}

The model assumes that the second follower has a prior belief about what the first offer is and denotes the density and distribution of this prior by $g(\cdot)$ and $G(\cdot)$ respectively. We assume that $G(\cdot)$ is normally distributed. The second follower has a noisy rational expectation in that $G(\cdot)$ has a mean of $s_{1}$ and a standard deviation of $\sigma_{1}$. Given the signal $z=s_{1}+\epsilon$, the second follower forms a posterior belief of the first offer, with density $h(\cdot)$, given by:

$$
h(x \mid z)=\frac{g(x) \cdot f(z-x)}{\int_{\infty}^{-\infty} g(x) \cdot f(z-x) d x} .
$$

The second follower anticipates that the first follower has an acceptance threshold $A$ satisfying $U_{F 1}\left(s_{1}=A, a_{1}=1\right)=0$, so the first follower will accept all offers $s_{1} \geq A$. Therefore, the second follower's inference of the probability $\hat{p}(z)$ that the first follower has accepted is

$$
\hat{p}(z)=\int_{A}^{\infty} h(x \mid z) d x .
$$

Similarly, the second follower's inference of the first offer, conditional on acceptance, is

model estimation yields an estimate $\hat{\eta}=0$. Also, a nested model restricting $\rho=\eta$ is strongly rejected $\left(\chi^{2}=108, p<0.01\right)$. Consequently, we did not include $\eta$ in our model to simplify exposition. 
given by:

$$
\hat{s_{1}}(z)=\frac{\int_{A}^{\infty} x \cdot h(x \mid z) d x}{\int_{A}^{\infty} h(x \mid z) d x} .
$$

We add an information inference process by the second follower for three reasons:

- This information inference process makes our model more realistic. In many reallife situations, the negotiation outcomes are kept confidential so as to avoid social comparison (e.g., employees are told not to reveal their raises to their peers). By allowing for imperfect information, we make our model applicable to more social settings.

- By introducing a noisy signal, we allow the leader to change his behavior as a result of the signal realization. Had the second follower perfectly known the first follower's payoff, the leader's offers in the two games would have been the same in equilibrium. Hence, imperfect information provides an extra degree of freedom to test the model. For instance, we later show that the equilibrium second offer is always higher than the equilibrium first offer.

- The game with imperfect information also allows us to separate two fundamentally different kinds of peer-induced fairness from the leader's perspective. The leader may inherently want to treat both followers the same way (e.g., parents showing no favoritism among their children). ${ }^{6}$ In contrast, the leader may care about treating the two followers the same way only to the extent that the second follower is averse to being behind. In the former, the leader will divide the pie the same way in the two games independent of the signal. If the latter is true, the leader will in fact choose the second offer contingent on the second follower's belief of what the first follower has received (the higher the belief, the higher the offer).

\section{Equilibrium Analysis}

We work backward to derive the equilibrium predictions. In the second game, the leader makes an offer $s_{2}$ to the second follower, who then decides whether to accept or reject it. Recall that the signal of the first offer is $z$ and the utility function of the second follower is:

$$
U_{F 2}\left(s_{2}, a_{2} \mid z\right)=\left\{\begin{array}{cc}
s_{2}-\delta \cdot \max \left\{0,\left(\pi-s_{2}\right)-s_{2}\right\} & \\
-\rho \cdot \hat{p}(z) \cdot \max \left\{0, \hat{s_{1}}(z)-s_{2}\right\} & \text { if } a_{2}=1, \\
0, & \text { if } a_{2}=0
\end{array}\right.
$$

Thus, the second follower accepts the offer $s_{2}$ if and only if $U_{F 2}\left(s_{2}, 1 \mid z\right) \geq 0$. The leader's utility function is

$$
U_{L, I I}\left(s_{2}, a_{2} \mid z\right)= \begin{cases}\pi-s_{2}-\delta \cdot \max \left\{0, s_{2}-\left(\pi-s_{2}\right)\right\}, & \text { if } a_{2}=1 \\ 0, & \text { if } a_{2}=0\end{cases}
$$

\footnotetext{
${ }^{6}$ One can extend the basic model by allowing the leader to have an intrinsic preference for treating the two followers identically. This can be accomplished by adding an extra term $-\beta \cdot\left(\left|s_{1}-s_{2}\right|\right)$ to the leader's utility function. However, our experimental data indicates that the leader tends to choose the second offer based on the second follower's inference of what the first follower has received, which may be different from the actual first offer. Such strategic behavior suggests that the leader does not have a strong intrinsic preference to treat the two followers the same way.
} 
Since the leader's utility $\left.U_{L, I I}\left(s_{2}, 1 \mid z\right)\right)$ always decreases in $s_{2}$, the leader will want to choose the smallest acceptable offer $s_{2}$ satisfying $U_{F 2}\left(s_{2}, 1 \mid z\right) \geq 0$. The following proposition characterizes the optimal offer $s_{2}^{*}$.

PROPOSITION 1: The leader's optimal offer to the second follower $s_{2}^{*}$, as a function of the follower's inferences $\hat{p}(z)$ and $\hat{s_{1}}(z)$, is

$$
s_{2}^{*}\left(\hat{p}(z), \hat{s_{1}}(z)\right)=\min \left\{\max \left\{\frac{\pi \cdot \delta}{1+2 \cdot \delta}, \frac{\pi \cdot \delta+\rho \cdot \hat{p}(z) \cdot \hat{s_{1}}(z)}{1+2 \cdot \delta+\rho \cdot \hat{p}(z)}, \frac{\rho \cdot \hat{p}(z) \cdot \hat{s_{1}}(z)}{1+\rho \cdot \hat{p}(z)}\right\}, \frac{\pi(1+\delta)}{1+2 \delta}\right\} .
$$

Proof: See Appendix.

Note that the optimal offer is the minimum of two terms: 1) $\max \left\{\frac{\pi \cdot \delta}{1+2 \cdot \delta}, \frac{\pi \cdot \delta+\rho \cdot \hat{p}(z) \cdot \hat{s}_{1}(z)}{1+2 \cdot \delta+\rho \cdot \hat{p}(z)}\right.$, $\left.\frac{\rho \cdot \hat{p}(z) \cdot \hat{s_{1}}(z)}{1+\rho \cdot \hat{p}(z)}\right\}$ and 2) $\frac{\pi(1+\delta)}{1+2 \delta}$. The first term yields the leader's most preferred offer while satisfying the incentive compatibility constraint (i.e., it is the smallest offer that induces the second follower to accept). The second term provides an upper bound of the second offer beyond which the leader will make a negative utility (due to distributional fairness concerns). The first term is determined by taking the maximum of three fractions. Note that the first fraction is independent of $\rho$ and the third fraction is independent of $\delta$. Consequently, the first/third fraction becomes relevant when distributional fairness/ peer-induced fairness is dominant. The second fraction comes into play when both kinds of fairness are of comparable magnitude.

Proposition 1 highlights that the equilibrium offer $s_{2}^{*}$ in the second game is nondecreasing in the second follower's inference $\hat{s_{1}}(z)$. In fact, when $\hat{s_{1}}(z)$ is sufficiently large, $s_{2}^{*}$ is strictly increasing in $\hat{s_{1}}(z)$ in a piecewise linear manner. This provides a sharp prediction on the leader's behavior. If the second follower has peer-induced fairness concerns (i.e., $\rho>0$ ) and the leader strategically anticipates such preferences, the leader should make the offer contingent on the inference $\hat{s_{1}}(z)$.

In the first game, the leader makes the offer $s_{1}$ to the first follower. Recall that the first follower's utility function is

$$
U_{F 1}\left(s_{1}, a_{1}\right)= \begin{cases}s_{1}-\delta \cdot \max \left\{0,\left(\pi-s_{1}\right)-s_{1}\right\}, & \text { if } a_{1}=1 \\ 0, & \text { if } a_{1}=0\end{cases}
$$

Therefore, the first follower accepts the offer $s_{1}$ if and only if $U_{F 1}\left(s_{1}, 1\right) \geq 0$, which can be shown to be equivalent to $s_{1} \geq \frac{\pi \cdot \delta}{1+2 \cdot \delta}$. In other words, the first follower's acceptance threshold is $A=\frac{\pi \cdot \delta}{1+2 \cdot \delta}$.

How much should the leader offer to the first follower? This decision influences the leader's material payoffs in both the first and the second games. Conditional on $s_{1}$ in the first game and along the equilibrium path in the second, the term $U_{L, I I}\left(s_{2}, a_{2} \mid z\right)$ can be written in terms of the signal $z$ as

$$
U_{L, I I}^{*}(z)=U_{L, I I}\left(s_{2}^{*}(z), a_{2}^{*}(z) \mid z\right) .
$$


Since the signal $z=s_{1}+\epsilon$, the expected value of the above utility given a first offer $s_{1}$ is

$$
E U_{L, I I}^{*}\left(s_{1}\right)=\int_{-\infty}^{\infty} U_{L, I I}^{*}\left(s_{1}+\epsilon\right) d F(\epsilon)
$$

Therefore, the leader chooses the first offer $s_{1}$ to maximize $U_{L, I}\left(s_{1}, a_{1}\right)+E U_{L, I I}^{*}\left(s_{1}\right)$. The following lemma states the relationship between the first offer $s_{1}$ and the leader's total expected utility in the second game, along the equilibrium path.

LEMMA 2: Condition on $s_{1}$ and along the equilibrium path, the leader's total expected utility in the second game, $E U_{L, I I}^{*}\left(s_{1}\right)$, is decreasing in $s_{1}$.

Proof: See Appendix.

The above lemma suggests that the leader incurs two costs in making a high offer $s_{1}$. First, a high $s_{1}$ will lower the leader's material payoffs in the first game. Second, this same high offer also leads to a lower expected utility for the leader in the second game. This is because a high $s_{1}$ sets a high reference point for social comparison by the second follower and this peer-induced fairness effect forces the leader to make a more generous offer $s_{2}$. Consequently, the leader will make the smallest possible offer $s_{1}$ in the first game. This offer is however constrained by the first follower's distributional fairness concerns. The following proposition states this result formally.

PROPOSITION 3: The leader's optimal offer to the first follower $s_{1}^{*}$ is

$$
s_{1}^{*}=\frac{\pi \cdot \delta}{1+2 \cdot \delta} .
$$

Proof: See Appendix.

As a consequence of Proposition 3, we have the following corollary.

COROLLARY 4: Under all signal realizations, the leader always gives a higher offer (weakly) to the second follower, i.e. $s_{2}^{*} \geq s_{1}^{*}$.

Proof: See Appendix.

Let us consider a numerical example. Let $\delta=0.5, \rho=1.5, \pi=100$. Assume that the noise term $\epsilon$ is uniformly distributed over $\{-20,-10,0,10,20\}$ and the second follower's prior belief of the first offer is normally distributed with mean $s_{1}^{*}$ and variance $\sigma_{1}=20 .^{7}$ With these parameters, the equilibrium first offer is $s_{1}^{*}=25$. Given the offer, the first follower will accept (i.e., $a_{1}^{*}=1$ ). The possible signal values are $\{5,15,25,35,45\}$. The equilibrium second offers conditional on the signal are given in Table 1 below.

The second follower always accepts the offer at equilibrium. In this example, note the following about the relationship between the second offer and the signal:

1) The second offer $s_{2}^{*}$ is non-decreasing in the signal. At the highest possible signal, the offer is about 20 percent above the first offer $s_{1}^{*}$.

\footnotetext{
${ }^{7}$ We chose these parameters and assumptions because they are close to our structural estimates and experimental setup described below.
} 


\begin{tabular}{cc}
\hline Signal $(z)$ & Equilibrium Second Offer $\left(s_{2}^{*}\right)$ \\
\hline \hline 5 & 25 \\
\hline 15 & 26.30 \\
\hline 25 & 27.69 \\
\hline 35 & 28.96 \\
\hline 45 & 30.25 \\
\hline
\end{tabular}

Table 1: Equilibrium Second Offers in a Numerical Example

2) The second offer $s_{2}^{*}$ is always greater than the first offer $s_{1}^{*}=25$, a constraint imposed by distributional fairness concerns. This result suggests that the leader is more generous to the second follower.

\section{A Variant with Simultaneous Offers}

In this subsection, we consider a simultaneous analog of the above model. As before, the leader has two separate pies to divide, each with a separate follower. However, the difference is that the leader now makes offers to both followers simultaneously. The game begins with the leader making offers $s_{i}$ to follower $i=1,2$. Each follower $i$ may accept the offer $\left(a_{i}=1\right)$ or reject it $\left(a_{i}=0\right)$. If $a_{i}=1$, the leader receives $\pi-s_{i}$ and follower $i$ receives $s_{i}$; otherwise, both receive zero from the corresponding pie. Note that each follower's acceptance decision influences only the division of one of the two pies. ${ }^{8}$

Consistent with the earlier setup, each follower's offer is privately observed, but there is a signal inference process before the followers make their decision. Let $\Delta \equiv s_{2}-s_{1}$ denote the true difference between the two offers (that is known to the leader but not to either follower). Both followers observe a public signal of this difference $z=\Delta+\epsilon$, where $\epsilon$ is a zero-mean random noise term with distribution $F(\cdot)$. Like before, the followers have noisy rational expectations. Each follower's prior belief $G(\cdot)$ over the difference $\Delta$ is normally distributed with mean $\Delta$ and standard deviation $\sigma$. After each follower $i$ observes the signal $z$ and her own offer $s_{i}$, each follower forms a posterior belief over the other offer. Specifically, letting $m(z) \equiv E[\Delta \mid z]$, follower 1's posterior expectation is $E\left[s_{2} \mid z, s_{1}\right]=s_{1}+m(z)$ while follower 2's posterior expectation is $E\left[s_{1} \mid z, s_{2}\right]=s_{2}-m(z)$. Observe that $m(z)$ is the followers' common posterior belief of the difference between the two offers. When $m(z)>0$, both followers believe that the leader has made a higher offer to follower 2 , and vice versa.

Based on the signal $z$, each follower chooses whether to accept or reject. Let us first focus on follower 1. Follower 1's utility is

$$
s_{1}-\delta \cdot \max \left\{0, \pi-2 s_{1}\right\}-\rho \cdot \max \{0, m(z)\}
$$

\footnotetext{
${ }^{8}$ This is different from Fehr and Schmidt (1999) who consider multiple receivers responding to offers on the same pie.
} 
from accepting the offer and zero from rejecting it. Given the offer

$$
s_{1}=\frac{\delta \cdot \pi+\rho \cdot \bar{m}_{1}}{1+2 \delta}
$$

for some $\bar{m}_{1}>0$, follower 1 will accept it as long as $m(z) \leq \bar{m}_{1}$. In other words, the threshold $\bar{m}_{1}$ represents the largest unfavorable disparity in payoffs that follower 1 is willing to tolerate. Therefore, the above offer $s_{1}$ will be accepted with probability $P\left\{m(z) \leq \bar{m}_{1}\right\}$. Now, let us turn to follower 2 , whose utility is

$$
s_{2}-\delta \cdot \max \left\{0, \pi-2 s_{2}\right\}-\rho \cdot \max \{0,-m(z)\}
$$

from accepting and zero from rejecting. Similarly as above, if given the offer

$$
s_{2}=\frac{\delta \cdot \pi+\rho \cdot \bar{m}_{2}}{1+2 \delta}
$$

for some $\bar{m}_{2}>0$, follower 2 will accept it whenever $m(z) \geq-\bar{m}_{2}$, which occurs with probability $P\left\{m(z) \geq-\bar{m}_{2}\right\}$.

Anticipating the followers' response, the leader chooses the optimal offers $s_{1}^{*}, s_{2}^{*}$ to maximize expected payoff given by

$$
\left(\pi-s_{1}\right) \cdot P\left\{m(z) \leq \bar{m}_{1}\right\}+\left(\pi-s_{2}\right) \cdot P\left\{m(z) \geq-\bar{m}_{2}\right\} .
$$

In equilibrium, there are three possible scenarios, depending on the signal realization $z$. When $m(z)<-\bar{m}_{2}$, only follower 1 accepts. When $-\bar{m}_{2} \leq m(z) \leq \bar{m}_{1}$, both offers are accepted. When $m(z)>\bar{m}_{1}$, only follower 2 accepts.

We experimentally test the sequential game rather than the simultaneous game for 3 reasons. First, the sequential game is probably more common in practice (e.g., pattern bargaining). Second, the sequential setup is richer for model estimation. Since the leader observes the signal realization before making the second offer, she can choose the second offer based on the signal. Third, the sequential game allows us to separate whether the leader herself genuinely cares about treating the followers the same way or if she strategically responds to followers having peer-induced fairness. If the former is true, $s_{2}^{*}=s_{1}^{*}$. If the latter is true, $s_{2}^{*}>s_{1}^{*}$.

\section{Experimental Procedure}

Seventy-five undergraduate students at a western university participated in the experiment. ${ }^{9}$ There were four experimental sessions. Each session had between 15 and 21 subjects and always consisted of 24 decision rounds. Each subject played the game 24 times. The matching protocol was such that subjects were randomly matched with others in each round and they never knew the identities of other players. Each session lasted

\footnotetext{
${ }^{9}$ It is common to use undergraduates to test theories of industrial organization (see Charles A. Holt, 1995). The results could in principle be replicated with managers. Several previous studies comparing professionals and students find little difference between the two groups (see Charles R. Plott, 1987, and Sheryl B. Ball and Paula-Ann Cech 1996). Alternatively, one could use student subjects with different levels of experience with the task to assess whether experts behave differently from novices (e.g., Yun J. Jung, John H. Kagel, and Dan Levin, 1994).
} 
for one and a half hours. Subjects were paid a show-up fee of $\$ 5$ for arriving on-time and earned an average payment of about $\$ 19$. Before the experiment began, subjects were read the instructions aloud and were given a chance to ask questions in private. A copy of the instructions is given in Appendix B. The entire experiment was computerized to facilitate information passing and random matching.

We simplified the decision task as much as possible. For example, the instructions provided a table that shows the possible first offers corresponding to a given signal value. The anonymous subject matching procedure was intended to avoid communication between subjects. Since a random matching protocol is used in each round, we controlled for collusion, reciprocity, and reputation building behaviors. Therefore, each round could be framed as a one-shot game with new partners. In each round, subjects were randomly grouped in triplets. In each triplet, the three subjects were randomly assigned the roles of RED (leader), BLUE1 (the first follower), or BLUE2 (the second follower). ${ }^{10}$ The three players played two independent ultimatum games, each with a pie size of 100 points, in sequence.

RED and BLUE1 played Game I first. RED moved first and chose the first offer $s_{1}$ (an integer between 0 and 100) at which she wished to divide the pie between herself and the first follower. The computer routed the information on $s_{1}$ to BLUE1. BLUE1 then decided whether or not to accept the offer. If BLUE1 chose to accept, RED and BLUE1 received the allocated amount accordingly. If BLUE1 rejected, both players earned 0 point.

To construct the signal $z$, we drew a number from a discrete uniform distribution over the set $\{-20,-10,0,10,20\}$ and added it to the first offer. Consequently, given a signal $z$, the subjects could infer what the first follower is likely to receive. To measure $\hat{s_{1}}(z)$, we asked BLUE2 to make a guess of what the first offer was and rewarded the player a modest sum of 10 points for making a correct guess. ${ }^{11}$

Finally, RED and BLUE2 played Game II. RED moved first and made an offer $s_{2}$ to BLUE2. BLUE2 could either accept or reject. If BLUE2 chose to accept, both players received payoffs as allocated. Otherwise, both received nothing. The outcomes, including whether BLUE2 guessed correctly, were revealed only at the end of each decision round comprising of both Games I and II. Each BLUE player received only the outcomes of her own game.

Each player's total point earnings for a decision round were recorded. Note that the leader received point earnings from both Games I and II. At the end of the session, point earnings for all rounds were summed up and redeemed for cash payment at the rate of $\$ 0.01$ per point (i.e., each ultimatum game involved dividing a pie of $\$ 1$ ).

\footnotetext{
${ }^{10}$ We chose a role switching design for 2 reasons. First, this design makes the average payoff of each subject similar (since every subject has an equal chance to be the leader). Second, we believe that such a design increases subjects' understanding of the game and hence reduces noise in the data.

${ }^{11}$ One of the reviewers remarked that the belief elicitation procedure itself could have changed the second follower's acceptance decision. To test for this conjecture, subjects in one session were not asked to guess the first offer before their acceptance decision. We analyzed subjects' decisions in this session separately and found the structural estimates from this session to be similar to those of the other three sessions. Consequently, we pool all the data in our structural estimation below.
} 


\section{Estimation}

\section{A. Basic Results}

Table 2 shows the basic results. Note that few offers are above 50 percent of the pie. Across the two games, less than 5 percent of the offers are within this range. The modal offer is between 30 percent and 35 percent for both games. Few offers are below 15 percent of the pie. No more than 3.5 percent of the offers fall into this range across the games. Hence the subgame perfect equilibrium prediction of a very low offer is strongly rejected. There is a clear pattern of a higher rate of rejection as the offer decreases. For example, there is no single offer in the range of 45 percent to 50 percent that was rejected, while the rate of rejection ranges from 25.9 percent to 31.7 percent when the offers are within the range of 25 percent to 30 percent. The overall results suggest that subjects are not purely self-interested. In general our results are comparable to those of prior studies except that the offers are slightly lower and followers tend to reject less frequently. The 4 experimental sessions produced 600 observations. There were two observations for which the leader made an offer of 100 (the entire pie) in either Game I or Game II. These data points were removed as outliers, so our dataset has a sample size of $N=598$.

\begin{tabular}{ccccc}
\hline \multicolumn{3}{c}{ Game I } & \multicolumn{2}{c}{ Game II } \\
\hline Offer Range & Offers (percent) & Rejected (percent) & Offers (percent) & Rejected (percent) \\
\hline \hline$>50$ & $29(4.8)$ & $0(0)$ & $28(4.7)$ & $0(0)$ \\
\hline 50 & $35(5.9)$ & $0(0)$ & $35(5.9)$ & $2(5.7)$ \\
\hline $45-49.5$ & $14(2.3)$ & $0(0)$ & $24(4.0)$ & $0(0)$ \\
\hline $40-44.5$ & $109(18.2)$ & $1(0.9)$ & $116(19.4)$ & $2(1.7)$ \\
\hline $35-39.5$ & $93(15.6)$ & $6(6.5)$ & $75(12.5)$ & $5(6.7)$ \\
\hline $30-34.5$ & $140(23.4)$ & $15(10.7)$ & $149(24.9)$ & $13(8.7)$ \\
\hline $25-29.5$ & $58(9.7)$ & $15(25.9)$ & $63(10.5)$ & $20(31.7)$ \\
\hline $20-24.5$ & $77(12.9)$ & $15(19.5)$ & $74(12.4)$ & $11(14.9)$ \\
\hline $15-19.5$ & $22(3.7)$ & $8(36.4)$ & $14(2.3)$ & $6(42.9)$ \\
\hline $10-14.5$ & $14(2.3)$ & $7(50.0)$ & $17(2.8)$ & $11(64.7)$ \\
\hline$<10$ & $7(1.2)$ & $6(85.7)$ & $3(0.5)$ & $2(66.7)$ \\
\hline \hline All & $598(100.0)$ & $73(12.2)$ & $598(100.0)$ & $72(12.0)$ \\
\hline
\end{tabular}

Table 2: The Distribution of Offers and the Rates of Rejection

We tested the data for time trends in the leader's offers as well as the followers' acceptance decisions. For the offers $s_{i}$, we specified the model $s_{i}(t)=\kappa_{0}+\kappa_{1} \cdot t$. Here, $s_{i}(t)$ denotes the leader's $i$-th offer ( $i=1$ or 2 ) in the $t$-th decision round, averaged over all subjects in the same session. In this model, $\kappa_{1}$ captures any possible time trend. Similarly, for the acceptance decisions $a_{i}$, we fitted the logistic regression $P\left(a_{i}(t)=1\right)=\frac{e^{\kappa_{0}+\kappa_{1} \cdot t}}{1+e^{\kappa_{0}+\kappa_{1} \cdot t}}$. We found no significant time trends (i.e., $\kappa_{1}$ is not statistically different from zero) for both acceptance decisions and the second offer. For the first offer, there was no time trend beyond round 2. All our results (e.g. parameter estimates) remain unchanged whether or not we include the first 2 rounds of data. In the following analysis, we assume no time trend. 


\section{B. Does Peer-Induced Fairness Exist?}

The central hypothesis of this paper is that the second follower has peer-induced fairness concerns. The second follower's utility function (equation 2) implies that, all things being equal, the second follower receives a lower utility if she believes she is behind the first follower. If the second follower makes decision errors (i.e., quantal-respond instead of best-respond), the second follower is less likely to accept an offer if the difference between $\hat{s}_{1}(z)$ and the offer $s_{2}$ is high. ${ }^{12}$ Table 3 below shows how the rate of rejection varies depending on whether the second follower believes she is ahead $\left(s_{2}-\hat{s_{1}}(z)>0\right)$, on par $\left(s_{2}-\hat{s_{1}}(z)=0\right)$, or behind $\left(s_{2}-\hat{s_{1}}(z)<0\right)$.

\begin{tabular}{|cc|cc|cc|}
\hline \multicolumn{2}{|c|}{ Being Ahead $\left(s_{2}-\hat{s_{1}}(z)>0\right)$} & \multicolumn{2}{|c|}{ On Par $\left(s_{2}-\hat{s_{1}}(z)=0\right)$} & \multicolumn{2}{c|}{ Being Behind $\left(s_{2}-\hat{s_{1}}(z)<0\right)$} \\
\hline $\mathrm{N}$ & Number of Rejections & $\mathrm{N}$ & Number of Rejections & $\mathrm{N}$ & Number of Rejections \\
\hline 165 & $6(3.6$ percent $)$ & 110 & $5(4.5$ percent $)$ & 179 & $42(23.5$ percent $)$ \\
\hline
\end{tabular}

Table 3: Different Rates of Rejection when Follower 2 is Ahead or Behind

The results are clear: The second follower rejects a lot more frequently when she is behind than otherwise (23.5 percent versus 4 percent). We test this formally by running a random effects logistic regression with BLUE2's decision $a_{2}$ against the second offer $s_{2}$ and how much it differs from BLUE2's guess (which is an estimate for $\hat{s_{1}}(z)$ ). Let superscripts $i$ and $t$ denote subject and decision round, and let $x^{+} \equiv \max \{x, 0\}$. Formally, we have:

$$
P\left(a_{2}^{i t}=1\right)=\frac{\exp \left\{\gamma_{0}^{i}+\gamma_{1} \cdot s_{2}^{i t}+\gamma_{2} \cdot\left({\hat{s_{1}}}^{i t}\left(z^{i t}\right)-s_{2}^{i t}\right)^{+}\right\}}{1+\exp \left\{\gamma_{0}^{i}+\gamma_{1} \cdot s_{2}^{i t}+\gamma_{2} \cdot\left({\hat{s_{1}}}^{i t}\left(z^{i t}\right)-s_{2}^{i t}\right)^{+}\right\}}
$$

where $\gamma_{0}^{i}$ are subject-specific random effects. If BLUE2 has peer-induced preferences, we would expect $\gamma_{2}$ to be negative. The estimation result shows that $\hat{\gamma_{2}}=-0.024(p$ value $=0.05$ ). This result suggests that the second follower may indeed be reluctant to accept an offer that is inferior to that of a peer. ${ }^{13}$ This finding also casts some doubt on the self-interested assumption and theories that ignore peer-induced fairness concerns.

It is possible that the first follower may also exhibit peer-induced fairness. The first follower may look ahead and anticipate what the second follower will receive in the future. If this is true, the first follower's expectation may influence her decision to accept. We check this conjecture by running a random effects logistic regression with BLUE1's decision $a_{1}$ against the first offer $s_{1}$ and how much it differs from the (anticipated) sec-

\footnotetext{
${ }^{12}$ It is interesting to check whether the second follower's inference $\hat{s_{1}}(z)$ is accurate. We regress the second follower's guess against the actual amount received by the first follower. Formally, we have $\hat{s_{1}}(z)=\omega_{0}+\omega_{1} \times a_{1} s_{1}$. The best fitted regression line yields $\hat{\omega}_{0}=25.4$ percent $\left(p\right.$-value $\left.<1 \times 10^{-16}\right)$ and $\hat{\omega}_{1}=0.26\left(p\right.$-value $\left.=4.1 \times 10^{-10}\right)$. This suggests that the second follower's inference is aligned with the first offer but exhibits some biases. They tend to over-estimate the first offer when it is less than 34 percent and under-estimate it when it is above 34 percent.

${ }^{13}$ Upon a reviewer's suggestion, we also test for the existence of peer-induced fairness by manipulating the notion of a peer systematically. We ran a control session by making the first follower no longer a peer for the second follower. We used the same sequential game setup except that we manipulated the degree of similarity of the followers' situations by using a random device to determine the leader's offer to the first follower so that the second follower perceived the first offer to be incomparable to the second offer. Our results reveal that the second follower's decision becomes independent of first follower's payoff and there is indeed no peer-induced fairness effect in the control session: $\hat{\gamma_{2}}=0.024(p$-value $=0.41)$. This finding shows that when the second follower does not perceive the first follower as a peer, the former does not exhibit peer-induced fairness concerns.
} 
VOL. NO.

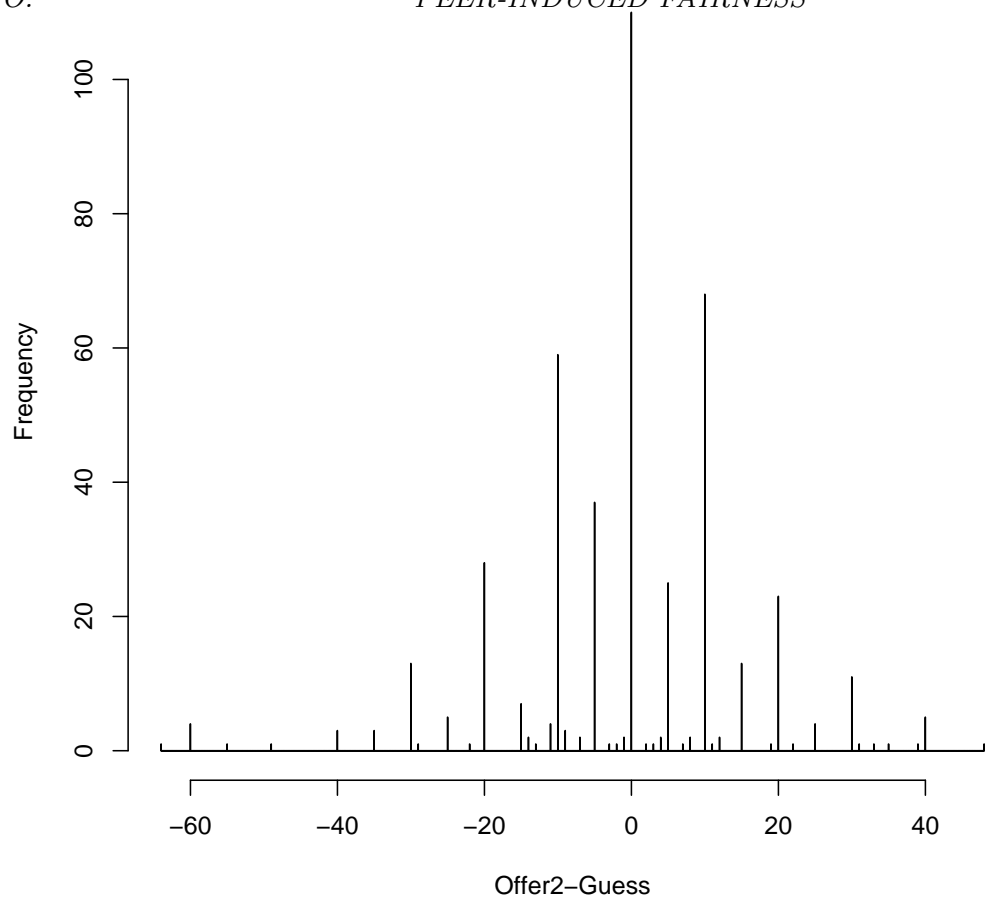

Figure 1: Observed Frequencies of $s_{2}-\hat{s_{1}}(z)$

ond offer $s_{2}$. Here, we assume that BLUE1 is able to predict the second offer perfectly. Formally, we have:

$$
P\left(a_{1}^{i t}=1\right)=\frac{\exp \left\{\gamma_{0}^{i}+\gamma_{1} \cdot s_{1}^{i t}+\gamma_{2} \cdot\left(s_{2}^{i t}-s_{1}^{i t}\right)^{+}\right\}}{1+\exp \left\{\gamma_{0}^{i}+\gamma_{1} \cdot s_{1}^{i t}+\gamma_{2} \cdot\left(s_{2}^{i t}-s_{1}^{i t}\right)^{+}\right\}}
$$

where $\gamma_{0}^{i}$ captures random effects. Like before, we would expect $\gamma_{2}$ to be negative and statistically different from zero. Our result indicates otherwise (the estimated $\hat{\gamma_{2}}=$ $-0.01<0, p$-value $=0.58)$.

\section{Did the Leader Respond to Peer-Induced Fairness?}

Proposition 1 suggests that the leader's offer in Game II is non-decreasing in $\hat{s_{1}}(z)$. Indeed, it is piecewise linear in $\hat{s_{1}}(z)$ if the latter is sufficiently high. Figure 1 shows the observed frequencies of the difference between the second offer and the guess, i.e. $\left(s_{2}-\hat{s_{1}}(z)\right)$. Note that this difference centers around zero and drops quickly as the difference gets larger suggesting that the offer may be influenced by the guess.

A simple test for this prediction is to regress $s_{2}$ against $\hat{s_{1}}(z)$. Formally, we have:

$$
s_{2}^{i t}=\alpha_{0}^{i}+\alpha_{1} \cdot{\hat{s_{1}}}^{i t}\left(z^{i t}\right) \text {, }
$$

where $\alpha_{0}^{i}$ are random effects. If the prediction is right, we expect $\alpha_{1}$ to be positive. The regression results suggest that $\alpha_{1}$ is indeed positive and statistically significant $\left(\hat{\alpha_{1}}=0.09\right.$ 
and $p$-value $=3.5 \times 10^{-3}$ ). This result implies that the leader is strategic and aligns her second offer with the second follower's inference $\hat{s_{1}}(z)$. As the second follower's inference increases, the leader who accounts for peer-induced fairness also strategically raises the second offer.

Furthermore, Corollary 4 suggests that by responding to peer-induced fairness concerns, the leader tends to be more generous to the second follower (i.e., $s_{2}^{*}>s_{1}^{*}$ ). We test this prediction using 2 methods. First, we treat each game outcome involving each triplet in a round as an independent observation. We perform a Wilcoxon signed-rank test with the null hypothesis that the medians of the distributions of the first and second offers are equal against the alternative hypothesis that the second is greater than the first. We use a one-sided test. There were $n=295$ observations for which the two offers were different. Under the null, the test statistic $W$ (sum of signed ranks) is normal with mean zero and standard deviation $\sqrt{\frac{n(n+1)(2 n+1)}{6}}=2933$. We obtain $W=5295$ ( $p$-value $=$ $0.03)$, and thus we can reject the null hypothesis. In the second method, we treat each subject's average offer across rounds as an independent observation. So, for each of the 75 subjects, we compute the average first offer and average second offer (across rounds). We then performed the one-tailed Wilcoxon test as before. The corresponding $p$-value is 0.04 , and thus we can again reject the null hypothesis. Therefore, we conclude that the second offer is indeed more generous (marginally) than the first offer.

\section{Parameter Estimation}

To formally estimate the relative importance of peer-induced and distributional fairness concerns, we structurally estimate the model parameters. The proposed model has two parameters, $\delta$ and $\rho$. The model involves 4 decisions, $s_{1}, s_{2}, a_{1}$, and $a_{2}$. We assume normal error terms $\xi_{1}, \xi_{2}$ for the leader's decisions,

$$
\begin{aligned}
& s_{1}=s_{1}^{*}+\xi_{1}, \\
& s_{2}=s_{2}^{*}+\xi_{2},
\end{aligned}
$$

so $s_{1}$ and $s_{2}$ have normal density $\phi_{1}(\cdot), \phi_{2}(\cdot)$ with means of $s_{1}^{*}$ and $s_{2}^{*}$ and variances of $\sigma_{1}^{2}$ and $\sigma_{2}^{2}$ respectively. The followers' utilities have an extreme value error term so that their acceptance probability has a logistic form with parameters $\lambda_{1}$ and $\lambda_{2}$ given below:

$$
\begin{aligned}
P_{1}\left(\delta, \lambda_{1}\right) & =\frac{e^{U_{F 1}(\delta) / \lambda_{1}}}{1+e^{U_{F 1}(\delta) / \lambda_{1}}} \\
P_{2}\left(\delta, \rho, \lambda_{2}\right) & =\frac{e^{U_{F 2}(\delta, \rho) / \lambda_{2}}}{1+e^{U_{F 2}(\delta, \rho) / \lambda_{2}}}
\end{aligned}
$$

In summary, the likelihood function for a set of decisions $s_{1}, s_{2}, a_{1}$, and $a_{2}$ is

$$
\phi_{1}\left(s_{1}\right) \cdot \phi_{2}\left(s_{2}\right) \cdot\left(P_{1}\right)^{a_{1}} \cdot\left(1-P_{1}\right)^{\left(1-a_{1}\right)} \cdot\left(P_{2}\right)^{a_{2}} \cdot\left(1-P_{2}\right)^{\left(1-a_{2}\right)},
$$

which we maximize over the parameters $\delta, \rho, \sigma_{1}, \sigma_{2}, \lambda_{1}, \lambda_{2}$.

Table 4 shows the estimation results. We estimate the full model and two nested models. The first column presents the nested model without any fairness concerns (i.e., $\delta=\rho=0$ or agents are purely self-interested). The second column gives the results when players have only distributional fairness concerns (i.e., $\rho=0$ ). The third column presents 
the full model. Both nested models are strongly rejected when compared to the full model indicating that subjects care about both distributional and peer-induced fairness. The self-interested hypothesis is clearly rejected $\left(\chi^{2}=1963.2, p\right.$-value $\left.<1.0 \times 10^{-16}\right)$. The nested model where the second follower has only distributional fairness is also strongly rejected $\left(\chi^{2}=108.0, p\right.$-value $\left.<1.0 \times 10^{-16}\right)$, suggesting that the second follower clearly has peer-induced fairness concerns. In the full model, the estimated peer-induced fairness parameter is $\hat{\rho}=1.746$, which is larger than the estimated distributional fairness parameter of $\hat{\delta}=0.501$. Given these parameter estimates and the logit specification in (21), the second follower's probability of rejection increases by 0.5 percent as her material payoff lags behind the leader's by one additional point (out of $\pi=100$ ). Analogously, the second follower's probability of rejection increases by 1.8 percent when her expected payoff difference behind the first follower increases by one point. These results suggest that peer-induced fairness (between followers) weighs more heavily than distributional fairness (between the leader and a follower) in the second follower's behavior.

\begin{tabular}{cccc}
\hline Parameter & No Fairness & Distributional Fairness Only & Full Model \\
\hline \hline$\delta$ & - & 0.597 & 0.501 \\
\hline$\rho$ & - & - & 1.746 \\
\hline$\lambda_{1}$ & 13.730 & 17.094 & 12.653 \\
\hline$\lambda_{2}$ & 14.139 & 16.688 & 24.702 \\
\hline$\sigma_{1}$ & 35.131 & 13.590 & 14.924 \\
\hline$\sigma_{2}$ & 35.017 & 12.895 & 11.082 \\
\hline \hline $\mathrm{LL}=$ & -6327.1 & -5408.5 & -5354.5 \\
\hline
\end{tabular}

Table 4: Estimation Results

\section{Incorporating Heterogeneity}

Our basic model adopts a representative-agent approach and assumes that all players have identical fairness concerns. In this section, we incorporate heterogeneity by analyzing a two-segment model in which one segment is purely self-interested and the other segment has both distributional and peer-induced fairness concerns. This extension is useful because a fraction of players is likely to be purely self-interested and we can then determine how self-interested players' behaviors are influenced by the existence of fairness-minded players.

In the two-segment model, let $\theta$ denote the fraction of the self-interested segment (i.e., the segment that has $\rho=\delta=0$ ). The remaining segment has distributional and peerinduced fairness concerns, represented by the parameters $\delta$ and $\rho$ as before. We shall derive the subgame perfect equilibrium using backward induction. The next proposition characterizes the leader's optimal offer $s_{2}^{*}$ in the second game. The key observation is that the leader may either make the same offer characterized in Proposition 1 (which induces both types to accept) or simply offer zero (in which case only the purely self-interested followers will accept). The former is preferred when the fraction of fairness-minded players is sufficiently large (i.e., $\theta$ sufficiently small). 
PROPOSITION 5: Suppose the follower's inference is $\hat{s_{1}}(z)$. Denote

$$
\tilde{s_{2}}=\min \left\{\max \left\{\frac{\pi \cdot \delta}{1+2 \cdot \delta}, \frac{\pi \cdot \delta+\rho \cdot \hat{p}(z) \cdot \hat{s_{1}}(z)}{1+2 \cdot \delta+\rho \cdot \hat{p}(z)}, \frac{\rho \cdot \hat{p}(z) \cdot \hat{s_{1}}(z)}{1+\rho \cdot \hat{p}(z)}\right\}, \frac{\pi(1+\delta)}{1+2 \delta}\right\} .
$$

The leader's optimal offer to the second follower is

$$
s_{2}^{*}= \begin{cases}\tilde{s_{2}}, & \text { if } \pi-\tilde{s_{2}}-\delta \cdot \max \left\{0,2 \tilde{s_{2}}-\pi\right\} \geq \theta \cdot \pi \\ 0, & \text { if } \pi-\tilde{s_{2}}-\delta \cdot \max \left\{0,2 \tilde{s_{2}}-\pi\right\}<\theta \cdot \pi\end{cases}
$$

Proof: See Appendix.

Next, consider the first game when there are both self-interested and fair-minded types. Similarly as above, the leader faces a choice between making the minimum acceptable offer to induce the fair-minded types to accept, and offering zero (in which case only the self-interested types will accept). As the next proposition shows, the former is preferred when the fraction of self-interested types $\theta$ is sufficiently small. The cutoff value for $\theta$ can be calculated numerically.

PROPOSITION 6: There exists some cutoff $\tilde{\theta} \in[0,1]$ such that the leader's optimal offer to the first follower is

$$
s_{1}^{*}= \begin{cases}\frac{\pi \cdot \delta}{1+2 \cdot \delta}, & \text { if } \theta \leq \tilde{\theta} \\ 0, & \text { if } \theta>\tilde{\theta}\end{cases}
$$

Proof: See Appendix.

We structurally estimate this two-segment model using the experimental data. ${ }^{14}$ This task helps to determine the fraction of the purely self-interested segment $\theta$. Table 5 shows the estimation results. The first column, for convenience, replicates the estimation results of the one-segment model, while the second column adds one additional parameter that represents the size of the purely self-interested segment $(\theta)$.

\footnotetext{
${ }^{14}$ Formally, we express the likelihood function for the data as follows. Let $\left(s_{1}^{i t}, s_{2}^{i t}, a_{1}^{i t}, a_{2}^{i t}\right)$ be the decisions made by subject $i$ in decision round $t$. Let $T_{L}^{i}, T_{F 1}^{i}, T_{F 2}^{i}$ denote the sets of decision rounds during which subject $i$ is the leader, follower 1 , and follower 2 , respectively. Given the model parameters $\theta, \delta, \rho, \sigma_{1}, \sigma_{2}, \lambda_{1}, \lambda_{2}$, the likelihood function for our data is

$$
\begin{gathered}
\prod_{i}\left[\theta \cdot\left(\prod_{t \in T_{L}^{i}} \phi_{1}^{0}\left(s_{1}^{i t}\right) \cdot \phi_{2}^{0}\left(s_{2}^{i t}\right) \cdot \prod_{t \in T_{F 1}^{i}}\left(P_{1}^{0}\right)^{a_{1}^{i t}} \cdot\left(1-P_{1}^{0}\right)^{\left(1-a_{1}^{i t}\right)} \cdot \prod_{t \in T_{F 2}^{i}}\left(P_{2}^{0}\right)^{a_{2}^{i t}} \cdot\left(1-P_{2}^{0}\right)^{\left(1-a_{2}^{i t}\right)}\right)\right. \\
\left(26 t(1-\theta) \cdot\left(\prod_{t \in T_{L}^{i}} \phi_{1}\left(s_{1}^{i t}\right) \cdot \phi_{2}\left(s_{2}^{i t}\right) \cdot \prod_{t \in T_{F 1}^{i}}\left(P_{1}\right)^{a_{1}^{i t}} \cdot\left(1-P_{1}\right)^{\left(1-a_{1}^{i t}\right)} \cdot \prod_{t \in T_{F 2}^{i}}\left(P_{2}\right)^{a_{2}^{i t}} \cdot\left(1-P_{2}\right)^{\left(1-a_{2}^{i t}\right)}\right)\right],
\end{gathered}
$$
}

where $\phi_{1}, \phi_{2}, P_{1}$ and $P_{2}$ are given as in (18) to (21), and $\phi_{1}^{0}, \phi_{2}^{0}, P_{1}^{0}$ and $P_{2}^{0}$ are defined similarly but with $\delta=\rho=0$. The expression in each pair of parentheses above represents the likelihood of observing subject $i$ 's decisions, given that subject $i$ is of a particular type (i.e., either self-interested or fair-minded) across all relevant decision rounds. In our structural estimation, we maximize the likelihood function (26) over the parameters $\theta, \delta, \rho, \sigma_{1}, \sigma_{2}, \lambda_{1}, \lambda_{2}$. 


\begin{tabular}{ccc}
\hline Parameter & Full Model (One segment) & Full model (Two segments) \\
\hline \hline$\delta$ & 0.501 & 0.771 \\
\hline$\rho$ & 1.746 & 1.619 \\
\hline$\lambda_{1}$ & 12.653 & 9.941 \\
\hline$\lambda_{2}$ & 24.702 & 10.033 \\
\hline$\sigma_{1}$ & 14.924 & 10.410 \\
\hline$\sigma_{2}$ & 11.082 & 10.821 \\
\hline \hline$\theta$ & - & 0.503 \\
\hline \hline $\mathrm{LL}=$ & -5354.5 & -4990.0 \\
\hline
\end{tabular}

Table 5: Estimation Results for Model Extensions

These results strongly suggest that there is substantial heterogeneity in subjects' preferences for fairness. About fifty percent of the subjects are estimated to be purely self-interested. Consequently, the representative-agent assumption is strongly rejected $\left(\chi^{2}=729.0, p\right.$-value $\left.<1.0 \times 10^{-16}\right)$. Furthermore, observe that the model estimates for the fair-minded segment are $\hat{\delta}=0.771$ and $\hat{\rho}=1.619$. Note that the degree of aversion to being behind a peer $(\rho)$ is 2 times stronger than the degree of aversion to being distributionally behind $(\delta)$. Given these parameter estimates, within the fair-minded segment, the second follower's probability of rejection increases by 1.9 percent (4.0 percent) as her material payoff lags behind the leader's (respectively, the first follower's) by one additional point. Similarly as before, these results suggest that peer-induced fairness concerns influence the second follower's actions more heavily than distributional fairness concerns.

\section{Economic Applications of Peer-Induced Fairness}

Many economic models can be substantially enriched by incorporating peer-induced fairness. In this section, we sketch three simple applications in which peer-induced fairness plays an important role. Specifically, we show how peer-induced fairness alone (without distributional fairness) can limit the degree of price discrimination, account for low variability in CEO compensation, and lead to the occurrence of labor strikes.

\section{A. Price Discrimination}

Many firms charge the same price in different markets even though the opportunity for price discrimination exists. Peer-induced fairness provides a plausible rational explanation for this phenomenon. Consider a monopoly selling in two separate markets $i=L, H$. Suppose that the demand function for each market is linear, with $D_{i}\left(p_{i}\right)=A_{i}-p_{i}$ for $i=L, H$. Equivalently, we can think of each market as a mass of $A_{i}$ consumers, whose valuations are uniformly distributed between 0 and $A_{i}$. Let $A_{L}<A_{H}$. In other words, we can think of Market L as the low-value market and Market $\mathrm{H}$ as the high-value market. The marginal production cost for both markets is denoted $c$. By the standard textbook analysis, we can calculate the monopoly's profit-maximizing price in each market to be $p_{i}^{*}=\left(A_{i}+c\right) / 2$. Under this result, the monopolist charges a higher price in the high-value market than in the low-value market.

Now, suppose that consumers have peer-induced fairness concerns. Similar to our model setup, assume that the monopolist enters the two markets sequentially. In this case, consumers in the late market will be averse to paying a higher price compared to 
consumers in the early market. Which market should the monopolist target first?

Consider first the case where the monopolist enters the high-value market before moving to the low-value market. Note that the profit-maximizing prices remain unchanged at $p_{i}^{*}=\left(A_{i}+c\right) / 2$. This is because the price $p_{H}^{*}$ for the high-value market sets a high reference point for social comparison. Consequently, consumers in the low-value market who face a lower price $p_{L}^{*}$ will not be affected by peer-induced fairness concerns.

However, the analysis changes dramatically when the monopolist first enters the lowvalue market. This initial price for the low-value market sets a reference point for consumers in the high-value market. ${ }^{15}$ When a consumer from the high-value market with valuation $v$ pays price $p_{H}$ for the product and the earlier price set for the low-value market is $p_{L}$, the consumer receives utility $v-p_{H}-\rho \cdot\left(p_{H}-p_{L}\right)$. Therefore, only consumers with valuations at least $p_{H}+\rho\left(p_{H}-p_{L}\right)$ are willing to buy. In other words, peer-induced fairness makes it more costly for the monopolist to raise price in the high-value market beyond that in the low-value market. It can then be shown that with peer-induced fairness concerns, the optimal prices satisfy $\left(A_{L}+c\right) / 2<p_{L}^{*} \leq p_{H}^{*}<\left(A_{H}+c\right) / 2$. Specifically, when $\rho$ is small enough, we have

$$
\begin{aligned}
p_{L}^{*} & =\frac{A_{L}+c+\frac{\rho}{2} \cdot\left(\frac{A_{H}}{1+\rho}-c\right)}{2-\frac{\rho^{2}}{2(1+\rho)}}>\frac{A_{L}+c}{2}, \\
p_{H}^{*} & =\frac{\frac{A_{H}+\rho \cdot p_{L}^{*}}{1+\rho}+c}{2}<\frac{A_{H}+c}{2} .
\end{aligned}
$$

However, when $\rho$ is sufficiently large, the monopolist prefers to eliminate price discrimination completely; this is done either by charging the same price $p_{L}^{*}=p_{H}^{*}=(\bar{A}+c) / 2$ in both markets, where $\bar{A}=\left(A_{L}+A_{H}\right) / 2$, if $A_{L}$ is large enough, or by simply forsaking the low-value market if $A_{L}$ is too small. The above analysis clearly indicates that the price differential over the two markets $p_{H}^{*}-p_{L}^{*}$ is smaller when there is peer-induced fairness. This discussion also suggests that the monopolist should first sell in the high-value market to maximize profit.

\section{B. Executive Compensation}

Why are CEO salaries so high? With the attractive executive remuneration packages in practice, the marginal utility gained from the last dollar in a CEO's pay is likely to be very small. That is, when the CEO's utility function $u(x)$ exhibits diminishing marginal utility, the marginal value of the $x$-th dollar $u^{\prime}(x)$ is very small when $x$ is very large. Since the CEO is not much worse off without that last dollar, why, then, are CEO salaries so high?

Peer-induced fairness concerns provide a possible explanation. Suppose that CEOs engage in social comparison with their peers, i.e. other CEOs. In this case, their utility function can be modeled as $v(x)=u(x)-\rho \max \{0, \hat{x}-x\}$, where $u(x)$ is the utility for money as above and $\hat{x}$ is the average compensation received by the focal set of CEOs.

\footnotetext{
${ }^{15}$ There are three possible market entry scenarios. The firm can either enter both markets simultaneously, enter the low-value market first, or enter the high-value market first. The analysis applies to the first two scenarios. It also applies to the third scenario if we allow consumers who make early purchases to look-ahead and make social comparison with consumers who make late purchases.
} 
Since individuals are likely to engage in upward social comparison by selecting individuals who are better as comparison benchmarks, we expect $\hat{x}>x$. Then, the marginal value of the $x$-th dollar is $v^{\prime}(x) \approx \rho$, which may be much higher than 0 (when $x$ is large, $u^{\prime}(x)$ is negligible). This discussion suggests that CEO remuneration packages are high not because of their material value, but because of the need to avoid discomforting social comparison (see also Charness and Peter Kuhn, 2004 for theory and evidence on wage compression and secrecy).

Peer-induced fairness also suggests that the reference or focal CEO set $(\hat{x})$ can significantly influence this social comparison process. For instance, O'Reilly, Main and Crystal (1988) show that there is a strong association between CEO compensation and the average compensation level of outside directors who serve on the compensation committee. This finding can be explained if CEOs treat members of the compensation committee as their peers.

\section{Union Negotiation}

In many industries, a large part of the workforce is represented by a nationally organized union which engages in pattern bargaining with multiple firms (Marshall and Merlo, 2004). Pattern bargaining consists of 3 features. First, the union chooses to negotiate with firms sequentially. Second, the union chooses the order with which it negotiates with firms. Third, the agreement with the first firm becomes the reference point that sets the pattern for all subsequent negotiations.

Suppose union $U$ and firm $F$ are negotiating over a pie, the size of which is normalized to one unit. Both $U$ and $F$ will receive the outside option of zero if they do not come to an agreement. If they do, let $x$ and $1-x$ be the shares of $U$ and $F$ respectively. Then, by standard analysis, it follows that for any $x \in(0,1)$, both parties will strictly prefer an agreement. In this case, we have $(0,1)$ as the feasible set.

Let the union's agreement with the first firm be $x^{\prime} \in(0,1)$. Now, consider a subsequent negotiation between the union and another firm. If the union (or its members) exhibit peer-induced fairness concerns, then in the current negotiation, $U$ 's utility from receiving $x$ will be $x-\rho \max \left\{0, x^{\prime}-x\right\}$. Observe that the feasible set of this game is now smaller, consisting only of allocations $x \in\left(\frac{\rho x^{\prime}}{1+\rho}, 1\right) \subset(0,1)$. That is, pattern bargaining reduces the feasible set of negotiation outcomes in the interests of the union. In addition, the union has an incentive to choose an order in which $x^{\prime}$ is maximized in the first firm.

Firms may also exhibit peer-induced fairness and bring to their respective negotiation their own comparison benchmarks. Let a specific firm's reference point be $x^{\prime \prime}$. In this case, $F$ 's utility from receiving $1-x$ will be $1-x-\rho \max \left\{0,\left(x-x^{\prime \prime}\right)\right\}$. We would expect $x^{\prime}>x^{\prime \prime}$ since each party's "comparable" outcome is likely to be biased in their own favor. For instance, Marc J. Knez and Camerer (1995) show experimentally that people apply different benchmarks for comparison when they have different outside options. Linda Babcock, Xianghong Wang, and Loewenstein (1996) provide empirical evidence for such a self-serving bias in teacher contract negotiations (see also Anand M. Goel and Anjan V. Thakor, 2005 for how optimal contract design could change as a result of peer-induced fairness effect and Werner Guth et. al, 2001 for a nice discussion on a similar issue). In this case, the feasible set of the game becomes $x \in\left(\frac{\rho x^{\prime}}{1+\rho}, \frac{1+\rho x^{\prime \prime}}{1+\rho}\right)$. In fact, when $x^{\prime}-x^{\prime \prime}>1 / \rho$, the feasible set is empty. This may occur when the two reference points diverge too widely (i.e., the gap $x^{\prime}-x^{\prime \prime}$ is too large), or when the degree of peer-induced fairness $\rho$ is 
large. This may explain why many labor contract negotiations end up in a strike. In most cases, there had been ample time and opportunities for interaction between negotiating parties. Peer-induced fairness suggests that an agreement is not feasible in the first place.

\section{Conclusions}

In this paper, we study peer-induced fairness in games. Peer-induced fairness concerns are prevalent because people have a natural tendency to look to their peers when evaluating their payoffs. This predisposition towards social comparison closely relates to the notions of conformism (George A. Akerlof, 1980 and B. Douglas Bernheim, 1984) and social influence (Chen Yan et al., 2007). We examine two distinct kinds of fairness concerns: 1) distributional fairness concerns (relative to other players in a game) and 2) peer-induced fairness concerns (relative to one's peers). Our work builds on that of Fehr and Schmidt (1999), which posits that economic agents experience a disutility when they receive a different material payoff compared to another reference agent or group.

We investigate peer-induced fairness in a sequence of two independent ultimatum games played by a leader and 2 followers. The leader plays an ultimatum game with the first follower, and then the same leader plays the same ultimatum game with the second follower. The games are independent in that each follower receives material payoff only in their respective game. Within each ultimatum game, the leader and the corresponding follower exhibit distributional fairness concerns in that both are averse to receiving less than each other. Between the two games, there is peer-induced fairness concerns in that the second follower is averse to receiving less than the first follower. In our model, the second follower does not perfectly observe what the first follower receives, but there is an information collection stage between the two games. That is, after the first ultimatum game, the second follower observes an imperfect signal of what the first follower is likely to receive before playing the second ultimatum game. We analyze the equilibrium of this game under imperfect information. Without peer-induced fairness, the second follower's acceptance decision and the leader's offer in the second game should not be influenced by the signal. In contrast, with peer-induced fairness, our model predicts that the second follower's behavior will be influenced by her inference of the first follower's payoff and that the leader will align the second offer with the second follower's inference.

We test our model predictions experimentally. Subjects are randomly assigned the roles of leader and followers and are motivated by financial incentives. We find strong support for our model predictions. Specifically, the second follower's rate of rejection increases with the difference between the second offer and her inference of the first follower's payoff. Also, the leader aligns the second offer close to the inference of the first follower's payoff in order to avoid rejection by the second follower. In combination, these results strongly suggest the existence of peer-induced fairness. We also structurally estimate our model using the experimental data. Our estimation results show that peer-induced fairness is distinct from distributional fairness and the former is crucial in explaining subjects' behaviors. The parameter estimates suggest that the second follower has a preference for peer-induced fairness that is 2 times stronger than her preference for distributional fairness (i.e., the former weighs more heavily in the second follower's decision).

We extend the basic model by allowing a fraction of the subjects to be purely selfinterested. Our structural estimation results indicate that about half of the subjects are purely self-interested while the other half exhibit fairness concerns. This result suggests 
that it is important to incorporate heterogeneity in the strategic analysis of games. Finally, we show how peer-induced fairness plays a key role in several economic applications. For example, peer-induced fairness can restrict a monopoly's ability to price discriminate, account for the low variability in CEO compensation, and lead to the occurrence of labor strikes.

\section{Appendix A: Proofs}

\section{Proof of Proposition 1}

The leader faces two alternatives. First, he may offer zero, which induces the follower to reject, and this leaves the leader with zero utility. Second, he may choose the optimal offer, among all the offers that are acceptable to the second follower. In other words, the leader solves the following problem:

$$
\begin{array}{cl}
\max _{s_{2}} & U_{L, I I}\left(s_{2}, 1 \mid z\right) \\
\text { s.t. } & U_{F 2}\left(s_{2}, 1 \mid z\right) \geq 0
\end{array}
$$

Note that this problem is equivalent to

$$
\begin{array}{cl}
\min _{s_{2}} & s_{2} \\
\text { s.t. } & U_{F 2}\left(s_{2}, 1 \mid z\right) \geq 0,
\end{array}
$$

since the leader's utility $U_{L, I I}\left(s_{2}, 1 \mid z\right)$ always increases as $s_{2}$ decreases. Introducing the variables $w_{1}=\max \left\{\pi-2 s_{2}, 0\right\}$ and $w_{2}=\max \left\{\hat{s_{1}}-s_{2}, 0\right\}$, we obtain the following problem. Denote the solution by $s_{2}^{0}$.

$$
\begin{array}{cl}
\min _{s_{2}, w_{1}, w_{2}} & s_{2} \\
\text { s.t. } & s_{2}-\delta \cdot w_{1}-\rho \cdot \hat{p} \cdot w_{2} \geq 0 \\
& w_{1} \geq \pi-2 s_{2} \\
& w_{2} \geq \hat{s_{1}}-s_{2} \\
& w_{1}, w_{2} \geq 0
\end{array}
$$

Notice that the above feasible region can be expressed in terms of only $s_{2}$ to yield:

$$
\begin{array}{ll}
\min _{s_{2}} & s_{2} \\
\text { s.t. } & s_{2}-\delta\left(\pi-2 s_{2}\right)-\rho \hat{p}\left(\hat{s_{1}}-s_{2}\right) \geq 0 \quad \Leftrightarrow s_{2} \geq \frac{\pi \delta+\rho \hat{p} \hat{s_{1}}}{1+2 \delta+\rho \hat{p}} \\
& s_{2}-\delta\left(\pi-2 s_{2}\right) \geq 0 \quad \Leftrightarrow s_{2} \geq \frac{\pi \delta}{1+2 \delta} \\
& s_{2}-\rho \hat{p}\left(\hat{s_{1}}-s_{2}\right) \geq 0 \quad \Leftrightarrow s_{2} \geq \frac{\rho \hat{p} \hat{s_{1}}}{1+\rho \hat{p}} \\
& s_{2} \geq 0
\end{array}
$$

Therefore, among all the offers that are acceptable to the second follower, the offer that maximizes the leader's utility $U_{L, I I}\left(s_{2}, a_{2} \mid z\right)$ is

$$
s_{2}^{0}=\max \left\{\frac{\pi \delta}{1+2 \delta}, \frac{\pi \delta+\rho \hat{p} \hat{s_{1}}}{1+2 \delta+\rho \hat{p}}, \frac{\rho \hat{p} \hat{s_{1}}}{1+\rho \hat{p}}, 0\right\} .
$$


Next, notice that the offer $s_{2}^{1}$ that leaves the leader with zero utility is

$$
s_{2}^{1}=\pi-\frac{\pi \delta}{1+2 \delta}=\frac{\pi(1+\delta)}{1+2 \delta} .
$$

Finally, we see that the leader's equilibrium offer in the second game must be $\min \left\{s_{2}^{0}, s_{2}^{1}\right\}$, as given in the proposition.

\section{Proof of LEMma 2}

Consider two possible offers $s_{1}$ and $s_{1}^{\prime}=s_{1}+k$ with $k>0$. Under the same noise term $\epsilon$, the signal realizations are $z=s_{1}+\epsilon$ and $z^{\prime}=s_{1}^{\prime}+\epsilon$ in the two cases. Note that they differ by $k$ exactly. Given the same noise term $\epsilon$, the posterior distribution $H^{\prime}$ of the first offer under true offer $s_{1}^{\prime}$, is thus a translation (to the right) of the posterior distribution $H$ of the first offer under true offer $s_{1}$. Let $\hat{p}, \hat{s_{1}}$ denote the inferences corresponding to true offer $s_{1}$, and let $\hat{p}^{\prime}, \hat{s}_{1}^{\prime}$ denote the inferences corresponding to true offer $s_{1}^{\prime}$, under some fixed noise term $\epsilon$. Note that $\hat{p}^{\prime}=\hat{p}+k h(A)+o(k)$ and $\hat{s}_{1}{ }^{\prime}=\hat{s}_{1}+k+k A h(A)+o(k)$. Recall $A=\frac{\delta \pi}{1+2 \delta}$ is the first follower's acceptance threshold.

To prove our result, we shall show that the equilibrium second offer satisfies $s_{2}^{*}\left(\hat{p}^{\prime}, \hat{s}_{1}^{\prime}\right) \geq$ $s_{2}^{*}\left(\hat{p}, \hat{s_{1}}\right)$. From Proposition 1, considering each individual term separately, it suffices to show $\frac{\pi \delta+\rho \hat{p}^{\prime} \hat{s}_{1}^{\prime}}{1+2 \delta+\rho \hat{p}^{\prime}} \geq \frac{\pi \delta+\rho \hat{p} \hat{s}_{1}}{1+2 \delta+\rho \hat{p}}$ and $\frac{\rho \hat{p}^{\prime} \hat{s}_{1}^{\prime}}{1+\rho \hat{p}^{\prime}} \geq \frac{\rho \hat{p} \hat{s}_{1}}{1+\rho \hat{p}}$.

For the first inequality above, we need to show

$$
\left[\pi \delta+\rho(\hat{p}+k h(A))\left(\hat{s_{1}}+k+k A h(a)\right)\right][1+2 \delta+\rho \hat{p}] \geq\left[\pi \delta+\rho \hat{p} \hat{s_{1}}\right][1+2 \delta+\rho(\hat{p}+k h(A))] .
$$

This inequality holds because we have $\rho k h(A) \hat{s_{1}}(1+2 \delta+\rho \hat{p}) \geq\left(\pi \delta+\rho \hat{p} \hat{s_{1}}\right) \rho k h(A)$, since $\hat{s_{1}} \geq \frac{\delta \pi}{1+2 \delta}$ as the first follower's acceptance threshold is $A=\frac{\delta \pi}{1+2 \delta}$. For the second inequality above, we need to show

$$
\rho[\hat{p}+k h(A)]\left[\hat{s_{1}}+k+k A h(A)\right][1+\rho \hat{p}] \geq \rho \hat{p} \hat{s_{1}}[1+\rho(\hat{p}+k h(A))] .
$$

This inequality holds because the right-hand-side exceeds $\rho \hat{p} \hat{s}_{1}(1+\rho \hat{p})$ by $\rho^{2} \hat{p} \hat{s}_{1} k h(A)$ but the left-hand-side exceeds by more.

Therefore, we have shown that for each noise term $\epsilon$, the equilibrium second offer is larger when the first offer is $s_{1}^{\prime}$ compared to $s_{1}$. The lemma thus follows.

\section{Proof of Proposition 3}

By Lemma 2, we know that $E U_{L, I I}^{*}\left(s_{1}\right)$ is decreasing in $s_{1}$. Also, note that $U_{L, I I}^{*}(z) \leq$ $U_{L, I}\left(\frac{\pi \delta}{1+2 \delta}, 1\right)$. This holds because for any $z, U_{L, I I}^{*}(z)=U_{L, I I}\left(s_{2}^{*}(z), a_{2}^{*}(z) \mid z\right) \leq U_{L, I I}\left(\frac{\pi \delta}{1+2 \delta}, 1 \mid z\right) \leq$ $U_{L, I}\left(\frac{\pi \delta}{1+2 \delta}, 1\right)$.

Now, we evaluate the two alternatives facing the leader: offer zero (and the first follower rejects) or offer the optimal acceptable offer (and the follower accepts). Recall that the leader wishes to maximize $U_{L, I}\left(s_{1}, a_{1}\right)+E U_{L, I I}^{*}\left(s_{1}\right)$. When the leader offers zero to the first follower, the first term is zero and the second term is at most $U_{L, I}\left(\frac{\pi \delta}{1+2 \delta}, 1\right)$. Alternatively, the leader may make an offer that is acceptable to the first follower. Recall that only offers $s_{1} \geq A=\frac{\pi \delta}{1+2 \delta}$ are acceptable. Since both $U_{L, I}\left(s_{1}, a_{1}\right)$ and $E U_{L, I I}^{*}\left(s_{1}\right)$ are decreasing in $s_{1}$, the leader's optimal offer that is acceptable to the follower is $s_{1}=\frac{\pi \delta}{1+2 \delta}$. 
In this case, the first term is $U_{L, I}\left(\frac{\pi \delta}{1+2 \delta}, 1\right)$ and the second term is non-negative. The proposition thus follows.

\section{Proof of Corollary 4}

This follows from comparing the results in Propositions 1 and 3.

\section{Proof of Proposition 5}

It is clear that $\tilde{s_{2}}$ is the minimum offer that is acceptable to the type with fairness concerns. The leader may either: (i) offer $\tilde{s_{2}}$ and receive $U_{L, I I}\left(\tilde{s_{2}}, 1\right)$, or (ii) offer 0 and receive $\pi$ with probability $\theta$ and 0 with probability $1-\theta$ (i.e., the expected utility is $\theta \pi$ ). The leader thus chooses the better alternative, as characterized in the proposition.

\section{Proof of Proposition 6}

Recall that the leader wishes to maximize $U_{L, I}\left(s_{1}, a_{1}\right)+U_{L, I I}\left(s_{2}, a_{2}\right)$, where $a_{1}$ and $a_{2}$ now refers to the acceptance decisions of the fair-minded types. Note from Proposition 5 that along the equilibrium path, we have $U_{L, I I}^{*}\left(s_{2}, a_{2}\right)=\max \left\{U_{L, I I}\left(\tilde{s_{2}}, 1\right), U_{L, I I}(0,0)\right\}=$ $\max \left\{U_{L, I I}\left(\tilde{s_{2}}, 1\right), \theta \pi\right\}$. Thus the reasoning in the proof of Lemma 2 continues to apply to the first term and thus Lemma 2 holds. Therefore, the only candidates for the first offer $s_{1}$ are 0 and $\frac{\pi \cdot \delta}{1+2 \cdot \delta}$. We will analyze the increase in the leader's utility when he offers $s_{1}=0$, compared to when he offers $s_{1}=\frac{\pi \cdot \delta}{1+2 \cdot \delta}$; in this proof, we term this his incremental utility.

In Game I, the leader's utility from offering $\frac{\pi \cdot \delta}{1+2 \cdot \delta}$ does not depend on $\theta$; however, the leader's utility from offering 0 , which is $\theta \pi$, has derivative $\pi$ with respect to $\theta$.

Next, consider the leader's incremental utility from Game II along the equilibrium path. When $s_{1}=0$, the leader's utility is $\max \left\{U_{L, I I}\left(\tilde{s_{2}}, 1 \mid s_{1}=0\right), \theta \pi\right\}$. When $s_{1}=\frac{\pi \cdot \delta}{1+2 \cdot \delta}$, the leader's utility is $\max \left\{U_{L, I I}\left(\tilde{s_{2}}, 1 \mid s_{1}=\frac{\pi \cdot \delta}{1+2 \cdot \delta}\right), \theta \pi\right\}$. In both cases, the first term does not depend on $\theta$ and the second term has derivative $\pi$ with respect to $\theta$. Therefore, the derivative of the incremental utility (i.e. the difference) with respect to $\theta$ must be at least $-\pi$.

Combining the two games, the derivative of the incremental utility with respect to $\theta$ must be non-negative. In other words, as $\theta$ increases, offering $s_{1}=0$ always becomes more attractive. The proposition thus follows.

\section{Appendix B: Instructions}

This is an experiment in economic decision making. The instructions are simple and if you follow them carefully and make good decisions, you could earn a considerable amount of money which will be paid to you in cash before you leave today. Different subjects may earn different amounts of cash. What you earn today depends partly on your decisions, partly on the decisions of others, and partly on chance.

The experiment consists of 24 decision making rounds. There are 21 subjects in this room. In each round, we will randomly group you into 7 triplets. In each round and in each triplet, one subject will be RED player and two subjects will be BLUE players (BLUE1 and BLUE2). You have an equal chance of playing the role of RED, BLUE1 or BLUE2 in each round. The decision making task of each player will be explained below. 
It is important that you do not look at the decisions of others, and that you do not talk, laugh or exclaim aloud during the experiment. You will be warned if you violate this rule the first time. If you violate this rule a second time, you will be asked to leave and you will not be paid. That is, your total earnings will be zero.

\section{Experimental procedure}

In each round, the decision making task occurs in 3 stages, namely, I, II, and III. Each RED player and the 2 matched BLUE players (BLUE1 and BLUE2) undertake the task as follows. Again the assignment of your role is determined randomly so that each person in the triplet has an equal chance of playing RED, BLUE1 or BLUE2.

In Stage I, RED and BLUE1 will have a pot of 100 points to divide between them (BLUE2 will sit still in this stage). RED will make an offer of OFFER1 (ranging from 0 to 100 points) to give it to BLUE1. After receiving the offer OFFER1, BLUE1 must decide whether or not to accept it. If BLUE1 accepts the offer, RED will receive 100 OFFER1 points and BLUE1 will receive OFFER1 points. However, if BLUE1 rejects the offer, both RED and BLUE1 will receive nothing in that decision making round. Note that the outcome of Stage I (i.e. whether BLUE1 accepts the offer) will only be revealed to RED at the end of Stage III.

In Stage II, we randomly draw a number from a set of 5 numbers: -20, -10, 0, 10, 20. That is, each number has an equal chance of being drawn. We call the drawn number X. We generate a signal called SIGNAL1 by adding X to OFFER1. We will use the number SIGNAL1 in Stage III. Note that each triplet involves a different independent draw in each decision round. However, each draw is always from the same set consisting of the same 5 numbers.

Let's consider two examples to see how this signal generation process works. If SIGNAL1 $=30$, then there are five possible scenarios:

\begin{tabular}{ccc}
\hline Offer 1 & $\mathrm{X}$ & Signal1 \\
\hline \hline 50 & -20 & 30 \\
\hline 40 & -10 & 30 \\
\hline 30 & 0 & 30 \\
\hline 20 & 10 & 30 \\
\hline 10 & 20 & 30 \\
\hline
\end{tabular}

Note that if SIGNAL1=30, OFFER1 can range from 10 to 50 depending on the value of the random number X.

Similarly, if SIGNAL1=70, we have the following five possible scenarios:

\begin{tabular}{ccc}
\hline Offer 1 & $\mathrm{X}$ & Signal1 \\
\hline \hline 90 & -20 & 70 \\
\hline 80 & -10 & 70 \\
\hline 70 & 0 & 70 \\
\hline 60 & 10 & 70 \\
\hline 50 & 20 & 70 \\
\hline
\end{tabular}


That is, OFFER 1 can range from 50 to 90 . Note that the above two examples are chosen purely for illustration purposes. In no way, the shown values are indicative of the optimal choices.

BLUE2 will guess what OFFER1 is. If BLUE2 guess correctly, he or she will receive a total of 10 points. If BLUE2 guess wrongly, he or she will receive nothing. Note that BLUE2's guess, and whether it is correct, will be revealed to RED and BLUE2 only at the end of Stage III.

In Stage III, RED and BLUE2 will have a pot of 100 points to divide between them (i.e., BLUE1 will sit still). Before RED makes her offer, both RED and BLUE2 will be informed of the value of SIGNAL1. Note that SIGNAL1 is generated by adding the random draw X described in Stage II to the OFFER1 made by RED to BLUE1 in Stage I. Then, RED will make an offer OFFER2 (ranging from 0 to 100 points) to give it to BLUE2. After receiving the offer OFFER2, BLUE2 must decide whether or not to accept the offer. If BLUE2 accepts the offer, RED will receive 100 - OFFER2 points and BLUE2 will receive OFFER2 points. However, if BLUE2 rejects the offer, both RED and BLUE2 will receive nothing in that decision round.

At the end of Stage III, the RED and both BLUE subjects will be informed of their respective decision outcomes and point earnings. The above decision task is repeated for 24 times. In each round, 7 triplets will be formed. Each player in the triplet will have an equal chance playing RED, BLUE1 or BLUE2.

\section{Payoffs}

Your dollar earnings for the experiments are determined as follows. First, we will sum up your total point earnings from all 24 rounds. Then we will multiply your point earnings by 0.01 . This is the amount you will be paid when you leave the experiment. Note that the more points you earn, the more money you will receive.

\section{REFERENCES}

Akerlof, George A. 1980. "A Theory of Social Custom, of Which Unemployment May be One Consequence." Quarterly Journal of Economics, 94(4): 749-775.

Akerlof, George A., and Janet Yellen. 1990. "The Fair Wage Effort Hypothesis and Unemployment." Quarterly Journal of Economics, 105(2): 255-283.

Babcock, Linda, Wang Xianghong, and George Loewenstein. 1996. "Choosing the Wrong Pond: Social Comparisons that Reflect a Self-Serving Bias." Quarterly Journal of Economics, 111(1): 1-19.

Ball, Sheryl B., and Paula-Ann Cech. 1996. "Subject pool choice and treatment effects in economic laboratory research." Research in Experimental Economics Vol. 6, 239-292. Greenwich, CT: JAI Press.

Bernheim, B. Douglas. 1994. "A Theory of Conformity." The Journal of Political Economy, 102(5): 841-877.

Bolton, Gary, and Axel Ockenfels. 2000. "ERC: A Theory of Equity, Reciprocity and Competition." American Economic Review, 90: 166-193.

Bosman, Ronald, and Frans van Winden. 2002. "Emotional Hazard in a Power-to-Take Experiment." Economic Journal, 112: 147-69. 
Camerer, Colin F. 2001. "Prospect Theory in the Wild: Evidence from the Field." In Choices, Values and Frames, ed. Kahneman, Daniel, and Amos Tversky. Cambridge: Cambridge University Press.

Camerer, Colin F., Joseph Henrich, Robert Boyd, Samuel Bowles, Ernst Fehr, Herbert Gintis, and Richard McElreath. 2001. "Cooperation, Reciprocity and Punishment in Fifteen Small-Scale Societies." American Economic Review, 91: 73-78.

Camerer, Colin F., George Loewenstein, and Matthew Rabin. 2003. Advances in Behavioral Economics. Princeton: Princeton University Press.

Charness, Gary, and Peter Kuhn. 2004. "Do co-workers' wages matter? Theory and evidence on wage secrecy, wage compression and effort." IZA discussion paper, No. 1417.

Charness, Gary, and Matthew Rabin. 2002. "Understanding Social Preferences with Simple Tests." Quarterly Journal of Economics, 117: 817-869.

Chen, Yan, F. Maxwell Harper, Joseph Konstan, and Sherry X. Li. 2007. "Social Comparisons and Contributions to Online Communities: A Field Experiment on Movie Lens." University of Michigan Working Paper.

Costa-Gomes, Miguel, and Klaus G. Zauner. 2001. "Ultimatum Bargaining Behavior in Israel, Japan, Slovenia, and the United States: A Social Utility Analysis." Games and Economic Behavior, 34: 238-269.

Fehr, Ernst, and Urst Fischbacher. 2002. "Why Social Preference Matter - The Impact of Non-selfish motives on competition, cooperation and incentives." The Economic Journal, 112(478): C1-C33.

Fehr, Ernst, and Simon Gachter. 2000. "Fairness and Retaliation: The Economics of Reciprocity." The Journal of Economic Perspectives, 14(3): 159-181.

Fehr, Ernst, and Klaus M. Schmidt. 1999. "A Theory of Fairness, Competition and Cooperation." Quarterly Journal of Economics, 114: 817-868.

Fehr, Ernst, Goerge Kirchsteiger, and Arno Riedl. 1993. "Does Fairness prevent Market Clearing?" Quarterly Journal of Economics, 108: 437-460.

Festinger, Leon. 1954. "A theory of social comparison processes." Human Relations, 7(2): 117-140.

Goel, Anand M., and Anjan V. Thakor. 2005. "Optimal Contracts When Agents Envy Each Other." Washington University Working Paper.

Guth, Werner, Manfred Konigstein, Judit Kovacs, and Eniko Zala-Mezo. 2001. "Fairness within firms: the case of one principal and many agents." Schmalenbach Business Review, 53: 82-101.

Henrich, Joseph. 2000. "Does Culture Matter in Economic Behavior? Ultimatum Game Bargaining among the Machiguenga of the Peruvian Amazon." American Economic Review, 90: 973-979.

Ho, Teck-Hua, and Juanjuan Zhang. 2008. "Designing Pricing Contracts for Boundedly Rational Customers: Does the Framing of the Fixed Fee Matter?" Management Science, 54(4): 686-700.

Ho, Teck-Hua, Noah Lim, and Colin F. Camerer. 2006a. "Modeling the Psychology of Consumer and Firm Behavior Using Behavioral Economics." Journal of Marketing Research, 43: 307-331.

Ho, Teck-Hua, Noah Lim, and Colin F. Camerer. 2006b. "How Psychological Should Economic and Marketing Models Be?" Journal of Marketing Research, 43: 341-344.

Holt, Charles A. 1995. "Industrial organization: A survey of laboratory results." In Handbook of Experimental Economics, ed. Kagel, John H., and Alvin E. Roth, 349-443. 
Princeton, NJ: Princeton University Press.

Jung, Yun J., John H. Kagel, and Dan Levin. 1994. "On the Existence of Predatory Pricing: An Experimental Study of Reputation and Entry Deterrence in the Chain-Store Game." The RAND Journal of Economics, 25(1): 72-93.

Kahneman, Daniel, and Amos Tversky. 1979. "Prospect Theory: An Analysis of Decision under Risk." Econometrica, 47(2): 263-292.

Knez, Marc J., and Colin F. Camerer. 1995. "Outside Options and Social Comparison in Three-Player Ultimatum Game Experiments." Games and Economic Behavior, 10: 65-94.

Levinson, Harold M. 1960. "Pattern Bargaining: A Case Study of the Automobile Workers." The Quarterly Journal of Economics, 74 (2): 296-317.

Lim, Noah, and Teck-Hua Ho. 2007. "Designing Price Contracts for Boundedly Rational Customers: Does the Number of Blocks Matter?" Marketing Science, 26(3): 312-326.

Luttmer, Erzo F. P. 2005. "Neighbors as Negatives: Relative Earnings and Well-Being." The Quarterly Journal of Economics, 120(3): 963-1002.

Marshall, Robert C., and Antonio Merlo. 2004. "Pattern Bargaining." International Economic Review, 45(1): 239-255.

O'Reilly, Charles A., Brian G. Main, and Graef S. Crystal. 1988. "CEO Compensation as Tournament and Social Comparison: A Tale of Two Theories." Administrative Science Quarterly, 33(2): 257-274.

Plott, Charles R. 1987. "Dimensions of parallelism: Some policy applications of experimental methods." In Laboratory Experimentation in Economics: Six Points of View, ed. Alvin E. Roth. New York: Cambridge University Press.

Rabin, Matthew. 1993. "Incorporating Fairness into Game Theory and Economics." The American Economic Review, 83(5): 1281-1302.

Rabin, Matthew. 1998. "Psychology and Economics." Journal of Economic Literature, 36(1): 11-46.

Roth, Alvin E., Vesna Prasnikar, Masahiro Okuno-Fujiwara, and Shmuel Zamir. 1991. "Bargaining and Market Behavior in Jerusalem, Ljubljana, Pittsburgh, and Tokyo: An Experimental Study." American Economic Review, 81: 1068-1095.

Slonim, Robert, and Alvin E. Roth. 1998. "Learning in High Stakes Ultimatum Games: An Experiment in the Slovak Republic." Econometrica, 66(3): 569-596. 